\title{
Influência da exposição a fumaça lateral do cigarro sobre 0 ganho de peso e o consumo alimentar de ratas gestantes: análise do peso e do comprimento dos filhotes ao nascimento
}

\author{
Influence of side stream cigarette smoke exposure on body weight gain and food consumption of \\ pregnant rats: analysis of weight and length at birth
}

Paulo Roberto Bezerra de Mello', Thelma Suely Okay², Clovis Botelho³

\section{RESUM0}

Objetivos: analisar o efeito do tabagismo sobre o ganho ponderal e o consumo alimentar de ratas durante a gestação, e o peso e comprimento dos filhotes ao nascimento. Métodos: foram estudadas 51 ratas (Wistar) do $2^{\circ}$ dia até o final da gestação, divididas em três grupos: Grupo F: 15 ratas expostas à fumaça de dois cigarros/animal/dia + ar comprimido ( $10 \mathrm{~L} /$ min); Grupo Ar: 18 ratas expostas ao ar comprimido $(10 \mathrm{~L} / \mathrm{min})$; Grupo C: 18 ratas não manipuladas e não expostas. Os parâmetros aferidos foram: peso das ratas e seu consumo alimentar e comprimento e peso dos filhotes ao nascer. Foi aplicado o teste de Lavene para verificação do comportamento da distribuição das variáveis numéricas, e para análises paramétricas utilizou-se o ANOVA ou teste $t$ de Student, conforme o caso. O nível de significância adotado foi $\mathrm{p}<0,05$. Resultados: as ratas expostas ao tabaco consumiram menos alimentos por dia [ $\mathrm{Grupo} \mathrm{F}=18,9( \pm 1,2)$ vs $\mathrm{Grupo} \mathrm{Ar}=21,7( \pm 1,6)$ vs Grupo $\mathrm{C}=24,2( \pm 1,7) ;(\mathrm{p}<0,05)]$ e apresentaram menor ganho ponderal ao final da gestação em relação às ratas expostas ao ar comprimido e ao grupo controle: peso no vigésimo dia de gestação: Grupo $\mathrm{F}=338,9 \mathrm{~g}( \pm 13,8)$ vs $\mathrm{Grupo} \mathrm{Ar}=352,3 \mathrm{~g}( \pm 15,9)$ vs Grupo $\mathrm{C}=366,3 \mathrm{~g}( \pm 13,1) ; \mathrm{p}<0,001)$. O peso dos filhotes ao nascimento [(Grupo $\mathrm{F}=5,5 \mathrm{~g}( \pm 0,3)$; $\mathrm{Grupo} \mathrm{Ar}=5,9 \mathrm{~g}( \pm 0,5)$; Grupo $\mathrm{C}=5,9 \mathrm{~g}( \pm 0,4)-\mathrm{p}<0,01$ ] e o comprimento ao nascer [Grupo $\mathrm{F}=6,8 \mathrm{~cm}( \pm 0,2) ; \operatorname{Grupo} \mathrm{Ar}=6,9 \mathrm{~cm}( \pm 0,2) ; \mathrm{Grupo} \mathrm{C}=6,9 \mathrm{~cm}$ $( \pm 0,1)-\mathrm{p}<0,05]$ foram significativamente menores no grupo de ratas expostas ao tabaco em relação aos outros dois grupos, que não diferiram entre si. Conclusões: a exposição ao tabaco reduziu o ganho ponderal e o consumo alimentar das ratas durante a gestação e produziu redução do peso e do comprimento dos filhotes ao nascimento.

PALAVRAS-CHAVE: Tabagismo; Gestação; Ganho de peso; Peso ao nascer; Retardo do crescimento fetal; Ratos

\section{ABSTRACT}

Purpose: to analyze the effect of cigarette smoke on weight gain and food consumption of female pregnant rats, as well as of their offspring's weight and length at birth Methods: Wistar rats were studied from the second day until the end of pregnancy. Fifty-one female rats were divided into three groups: Group F: 15 rats exposed to tobacco smoke ( 2 cigarettes/animal/day) plus air flush (10 L/min); Group Air: 18 rats exposed to air flush only $(10 \mathrm{~L} / \mathrm{min})$; Group C: 18 non-exposed, non-manipulated controls. Body weight was measured every 5 days and food consumption every seven days (expressed as medium consumption per day). Offspring weight and length were measured on the first day of life. The Lavene test was used to verify the behavior of numeric variable distribution and for parametric one-way ANOVA analysis and Student's $t$ test were used, according to the case. Results with $\mathrm{p}<0.05$ were considered to be statistically significant. Results: the rats of Group F consumed less food per day [Group F=18.9 $\mathrm{g}( \pm 1.2)$ vs Group Air=21.7 $\mathrm{g}( \pm 1,6)$ vs Group $\mathrm{C}=24.2 \mathrm{~g}( \pm 1,7) ;(\mathrm{p}<0,05)$ ], gained less weight during pregnancy than the animals of the air flush group and the control group: Group F=338.9 $\mathrm{g}( \pm 13.7)$ vs Group Air $=352.3 \mathrm{~g}( \pm 15,9)$ vs Group $\mathrm{C}=366.3 \mathrm{~g}( \pm 13.1)(\mathrm{p}<0.05)$. Pups birth weight and length were significantly smaller in the smoking group when compared to controls and to the air flush group, while these last two groups did not differ: Group F=5.5 g $( \pm 0.3)$ vs Group Air $=5.9 \mathrm{~g}( \pm 0,5)$ vs Group C=5.9 g ( \pm 0.4$)$ - $(\mathrm{p}<0,01)$; Group F=6.8 cm ( \pm 0.2$)$ vs Group Air=6.9 cm $( \pm 0,2)$ vs Group C=6.9 cm ( \pm 0.1$)-(\mathrm{p}<0.05)$, respectively. Conclusions: tobacco smoke exposure reduced the weight gain and food consumption during pregnancy and reduced the offspring weight and length evaluated at birth.

KEYWORDS: Smoking; Pregnancy; Weight gain; Birth weight; Fetal growth retardation; Rats

\footnotetext{
Trabalho realizado na Faculdade de Ciências Médicas da Universidade Federal de Mato Grosso - UFMT - Cuiabá (MT), Brasil (Experimentos) e no Departamento de Pediatria da Faculdade de Medicina da Universidade de São Paulo - USP - São Paulo (SP), Brasil.

1 Professor Doutor do Departamento de Pediatria da Faculdade de Ciências Médicas da Universidade Federal de Mato Grosso - UFMT - Cuiabá (MT), Brasil.

2 Professora Doutora do Departamento de Pediatria da Faculdade de Medicina da Universidade de São Paulo - USP - São Paulo (SP), Brasil.

3 Professor Titular do Departamento de Clinica Médica/FCM e do Instituto de Saúde Coletiva da Universidade Federal de Mato Grosso - UFMT - Cuiabá (MT), Brasil.

Correspondência: Clovis Botelho

Rua Dr. Jonas Correa da Costa, 210 - 78.030-510 - Cuiabá - MT - Fone/Fax: (65) 3637-1471 - e-mail: fbotelho@terra.com.br

Recebido em: 24/2/2006 Aceito com modificacōes em: 28/3/2006
}

Rev Bras Ginecol Obstet. 2006; 28(3): 143-50 
Introdução

O tabagismo afeta todas as fases da reprodução humana, da gametogênese à lactação. Efeitos do tabagismo sobre o crescimento intra-uterino se manifestam de maneira mais visivel no terceiro trimestre da gravidez, e de forma dose-dependente ${ }^{1-3}$.

O tabagismo na gestação dobra a chance de ocorrência de baixo peso ao nascer, seja por restrição do crescimento intra-uterino (RCIU) seja por prematuridade $\mathrm{e}^{4,5}$. Mulheres que fumaram durante toda a gestação, ou que recomeçaram a fumar nos dois últimos trimestres, apresentaram o dobro de risco de RCIU em relação àquelas que fumaram apenas no primeiro e segundo trimestres $^{2}$.

Pesquisas realizadas em modelos animais observaram redução do ganho ponderal, ou até mesmo perda de peso após administração contínua de nicotina ${ }^{6,7}$. A maioria dos estudos mostra que o uso da nicotina produz período de perda de peso (ou redução do ganho de peso), assim como a cessação do uso da droga é seguida de período agudo de ganho de peso, com recuperação e equiparação ao peso observado nos animais controle. São três as teorias mais aceitas para explicar a relação do tabagismo e do peso corporal: aumento da taxa metabólica, com maior gasto de energia pelos fumantes ${ }^{8,9}$; diferenças na qualidade e quantidade dos alimentos ingeridos pelos fumantes ${ }^{10,11}$ e ação anorética da nicotina ${ }^{12,13}$.

Os mecanismos fisiopatológicos que levam ao retardo de crescimento intra-uterino em fetos de mães fumantes não estão totalmente elucidados ${ }^{14,15}$. Dentre os muitos constituintes da fumaça do cigarro, a nicotina e o monóxido de carbono têm sido considerados os principais responsáveis pelos efeitos deletérios no feto humano, visto que ambas as substâncias são rapidamente transferidas através da placenta ${ }^{16,17}$. A nicotina produz liberação de catecolaminas, com conseqüente vasoconstricção materna e fetal crônica, resultando em hipóxia fetal ${ }^{17,18}$.

A fumaça do cigarro liberada no meio ambiente é constituída por dois componentes: fumaça central ou principal e fumaça periférica ou lateral. A fumaça central é gerada quando o fumante traga o cigarro, é produzida em altas temperaturas (acima de $950^{\circ} \mathrm{C}$ ) e polui o ambiente após ter sido aspirada através do cigarro, entrado em contato com os pulmões e, em seguida, exala$\mathrm{da}^{19,20}$. Constitui a fonte predominante de exposição dos indivíduos fumantes ativos.
A fumaça periférica é produzida em temperaturas mais baixas (cerca de $350^{\circ} \mathrm{C}$ ), durante a queima lenta do cigarro que ocorre entre as tragadas. Oitenta e cinco por cento da fumaça de cigarro presente no ambiente é resultante da fumaça periférica produzida a partir da queima espontânea da extremidade do cigarro. Este componente difere da fumaça central inalada pelo tabagista ativo pelo fato de não ser filtrada, e a despeito de diferenças quantitativas, a composição química da fumaça lateral é similar à da fumaça principal ${ }^{19,21,22}$. A fumaça periférica é a fonte de exposição de todos aqueles que inalam a fumaça presente no meio ambiente, os chamados fumantes passivos.

Os fumantes passivos podem absorver até um sexto da quantidade de fumaça absorvida por um fumante ativo ${ }^{23}$. Mesmo que o risco para fumantes passivos seja menor do que para fumantes ativos, a proporção de pessoas fumantes passivas é muito maior ${ }^{24}$. Dentre os expostos de maneira passiva, aqueles que apresentam maior risco parecem ser os fetos ${ }^{23}$, recém-nascidos e lactentes jovens ${ }^{25}$. Metanálise que avaliou 25 trabalhos sobre o tema concluiu que a exposição passiva ao tabaco na gestação parece estar associada a discreta diminuição de peso ao nascimento e ao aumento no percentual de recém-nascidos com baixo peso ${ }^{26}$.

Em estudo realizado na Noruega, observouse que mães fumantes com niveis mais elevados de nicotina no cabelo apresentavam risco aumentado de terem filhos pequenos para a idade gestacional $^{27}$. Este efeito também foi observado em filhos de mães não fumantes, mas com niveis elevados de nicotina no cabelo, que apresentaram razão de chance de 3,4, indicando forte associação entre tabagismo passivo materno e o nascimento de crianças pequenas para idade gestacional.

A hipótese do presente estudo é que o tabagismo prejudica o ganho ponderal e o consumo alimentar de ratas gestantes causando crescimento intra-uterino restrito do feto. Para tornar o modelo de exposição mais próximo ao que ocorre na mulher grávida fumante passiva, foram substituídas as injeções de nicotina por aparato especialmente construído para fazer com que os animais inalassem fumaça proveniente da queima passiva de cigarros ${ }^{19,28}$. Assim, os objetivos do estudo foram: analisar o efeito do tabagismo sobre o ganho ponderal e o consumo alimentar de ratas gestantes e verificar o efeito do tabagismo sobre o peso e o comprimento dos filhotes ao nascimento. 


\section{Métodos}

Este estudo utilizou modelo experimental de exposição à fumaça lateral do tabaco desenvolvido em ratas nas quais vários parâmetros associados à gestação, ao bem-estar fetal e à lactação foram analisados ${ }^{28}$. Apenas os animais que completaram todo o ciclo reprodutivo, da gestação à lactação, foram considerados e as perdas desprezadas. Foram estudadas 51 ratas virgens de quatro meses de idade (Rattus norvegicus) da cepa Wistar fornecidas pelo Biotério Central da Universidade Federal de Mato Grosso e mantidas no Biotério Setorial do Laboratório de Investigação da Faculdade de Ciências Médicas da UFMT, em temperatura controlada $\left(22 \pm 2^{\circ} \mathrm{C}\right)$, umidade de 40 a $60 \%$ e ciclo de luz controlado (12 horas de claridade e 12 horas de escuridão). Os animais foram pesados e numerados por perfuração do pavilhão auricular à admissão. Foram aceitos animais com peso entre 230 e 260 g (balança analítica marca Marte A 500, com capacidade de $500 \mathrm{~g}$, e variação de 0,01 g), alimentados com ração Nuvilab (Nuvital, Curitiba, PR) e água ad libitum.

O ciclo estral das ratas foi avaliado durante 12 dias, por meio da análise da celularidade vaginal, realizada diariamente no horário entre 10 e 14 horas. No início de novo ciclo estral, cada rata foi colocada com um rato macho, e na manhã seguinte, a cópula foi confirmada pela presença de espermatozóides na vagina. O dia da cópula foi considerado como o dia zero da gestação, e as ratas foram pesadas na cópula e a cada cinco dias durante a gestação em balança analítica Marte A 500. Para corrigir o efeito do peso do animal no início do experimento, foram considerados também os valores da razão entre o peso de cada animal ao longo da gestação e o peso do animal no momento da cópula (peso proporcional).

A partir da cópula os animais foram divididos em 3 grupos: Grupo F (fumaça do tabaco): animais expostos à fumaça de 2 cigarros/animal/ dia, sob fluxo de ar comprimido em câmara de inalação (10 L/min), durante 15 minutos, duas vezes ao dia, durante toda gestação $(n=15)$; Grupo Ar (ar comprimido): animais expostos ao fluxo de ar comprimido (10 L/min), em câmara de inalação, durante 15 minutos, duas vezes ao dia, durante toda gestação $(n=18)$ e Grupo C (contro1e): animais que não sofreram exposição ou manipulação, exceto aquelas necessárias à pesagem $(n=18)$.
Para exposição dos animais à fumaça do tabaco foram utilizados cigarros da marca Marlboro (Phillip Moris), embalagem vermelha com 20 unidades. Cada cigarro contém $0,8 \mathrm{mg}$ de nicotina, $10 \mathrm{mg}$ de alcatrão e $10 \mathrm{mg}$ de monóxido de carbono (o rótulo do produto indica que estes valores foram aferidos, por amostragem, no Laboratório Labstat do Canadá).

O sistema de exposição utilizado consistiu de caixa retangular de madeira revestida de fórmica, medindo $94,5 \mathrm{~cm}$ de largura, $36,2 \mathrm{~cm}$ de altura e $27,5 \mathrm{~cm}$ de profundidade, dividida em dois compartimentos com portas individualizadas feitas em vidro: compartimento de combustão, medindo $42 \mathrm{~cm}$ de largura, e compartimento de inalação, medindo $52,5 \mathrm{~cm}$ de largura, separados entre si por uma divisória na qual existiam 4 orificios centrais com $1,5 \mathrm{~cm}$ de diâmetro ${ }^{29}$. Os animais foram trazidos do biotério anexo à sala de exposição, acomodados em pequenas gaiolas metálicas com três compartimentos cada, o que permitiu a exposição de até três animais por gaiola, que foram colocadas diretamente dentro do compartimento de inalação. O espaço do compartimento de inalação foi suficiente para acomodar até duas gaiolas por vez.

Foram realizados estudos preliminares com o intuito de verificar a eficácia da exposição à fumaça lateral de cigarros realizada com o protocolo e o aparato descrito ${ }^{30}$. Para tanto, as concentrações de carboxiemoglobina e de cotinina foram analisadas após exposição única, com modificação do número de cigarros queimados. Os coeficientes de correlação de Spearman, entre o número de cigarros e as concentrações de carboxiemoglobina e de cotinina, foram de 0,91 e 0,53 $(p<0,01)$, respectivamente. Para exposições prolongadas os mesmos coeficientes foram de 0,89 e de $0,75(p<0,01)$, respectivamente. Estes experimentos nortearam a escolha do número de cigarros/animal/dia e também o tempo de exposição (do $2^{\circ}$ dia até o final da gestação) e que corresponderia, aproximadamente, à concentração de nicotina presente em sangue humano após o consumo de no mínimo 7 cigarros em período de 24 horas.

Os animais do Grupo $\mathrm{F}$ (animais expostos ao tabaco) e Grupo Ar (ar comprimido) foram subdivididos em grupos de 5 animais cada, para serem submetidos à inalação por 15 minutos, duas vezes ao dia, com intervalo de 12 horas, a partir do $2^{\circ}$ dia até o final da gestação. Durante a exposição, os animais do Grupo F receberam a fumaça da queima passiva de 2 cigarros/animal/dia (5 cigarros por exposição). Os cigarros foram queima- 
dos completamente e ventilados por fluxo de ar comprimido de $10 \mathrm{~L} / \mathrm{min}$. Os animais do Grupo Ar utilizaram sistema de exposição exclusivo para este grupo, porém idêntico ao do Grupo F, no qual receberam somente o fluxo de ar comprimido (10 L/min), na mesma freqüência e duração, e nos mesmos horários.

O consumo alimentar foi medido por meio da pesagem da ração a cada 7 dias de gravidez, calculando-se a diferença entre a ração oferecida e a sobra acrescida das perdas recuperadas na limpeza do piso da gaiola e expresso como consumo alimentar médio do período gestacional.

O comprimento dos filhotes foi aferido pela medida nariz-extremidade da cauda do filhote estendido sobre uma superficie plana ${ }^{29}$. O comprimento e o peso ao nascimento foram obtidos nas primeiras 24 horas de vida dos animais.

A tabulação dos dados foi feita no programa Excel e as análises estatísticas com o programa SPSS (versão 9.0 for Windows). O teste de Lavene foi utilizado para verificação do comportamento da distribuição das variáveis numéricas. A seguir, os testes $t$ de Student ou ANOVA foram empregados, conforme o caso. O nível de significância adotado foi $\mathrm{p}<0,05$. O projeto de pesquisa foi aprovado pela Comissão para Análise de Projetos de Pesquisa - CAPPesq da Diretoria Clínica do Hospital das Clínicas da Faculdade de Medicina da Universidade de São Paulo (protocolo $\mathrm{n}^{\circ} 777 / 01$ ).

\section{Resultados}

Os resultados das medidas de peso das ratas na gestação (Figura 1) mostraram que os animais foram admitidos com pesos semelhantes e não mostraram diferença entre os pesos até o momento da cópula. Durante toda a gestação, o ganho ponderal foi significativamente diminuído no grupo de animais expostos à fumaça do tabaco, ao passo que os expostos ao ar comprimido tiveram peso intermediário entre os outros dois grupos ao final da gestação (peso no vigésimo dia de gestação: Grupo $\mathrm{F}=338,9 \mathrm{~g}( \pm 13,8)$ vs Grupo Ar=352,3 g $( \pm 15,9)$ vs Grupo $\mathrm{C}=366,3 \mathrm{~g}$ $( \pm 13,1) ; p<0,001$.

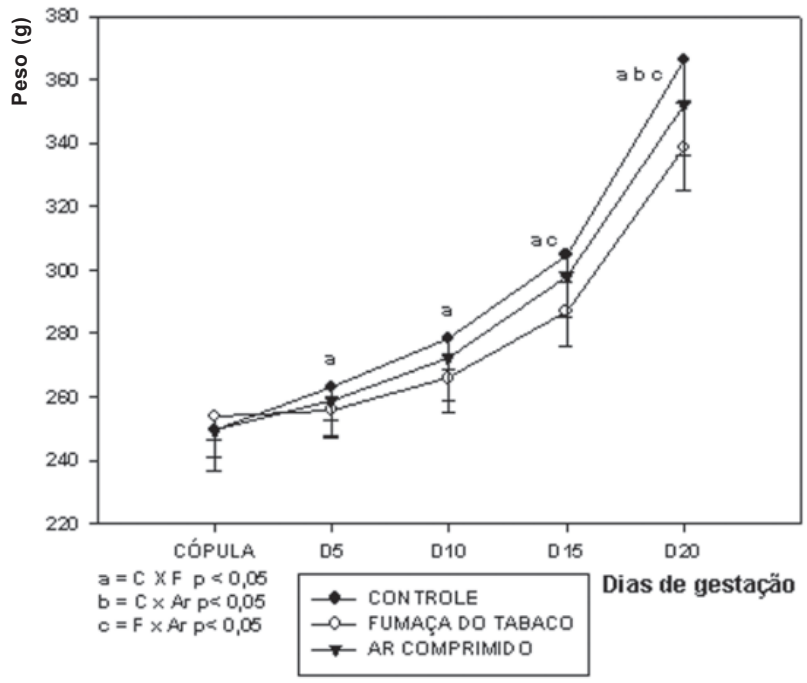

Figura 1 - Peso médio (g) na cópula e ao longo da gestação, obtido em intervalos de cinco dias, de ratas expostas à fumaça do tabaco $(\mathrm{F})$ e ao ar comprimido (Ar), comparadas a controles (C). D5 - quinto dia; D10 - décimo dia; D15 - décimo-quinto dia; D20 vigésimo dia.

Quando os valores de peso ao longo da gestação foram divididos individualmente pelo peso no momento da cópula (peso proporcional) (Figura 2), observou-se que em todos os tempos, as ratas do Grupo F apresentaram peso proporcional significativamente menor que os animais controle e os expostos ao ar comprimido $(\mathrm{p}<0,001)$. De forma semelhante, os animais expostos ao ar comprimido apresentaram ganho de peso proporcional significativamente menor durante a gestação, em relação aos animais do grupo controle $(\mathrm{p}<0,05)$. Peso proporcional no vigésimo dia de gestação: Grupo $\mathrm{F}=1,33( \pm 0,03)$ vs Grupo $\mathrm{Ar}=1,41( \pm 0,03)$ vs Grupo $\mathrm{C}=1,47( \pm 0,06) ; \mathrm{p}<0,001$.

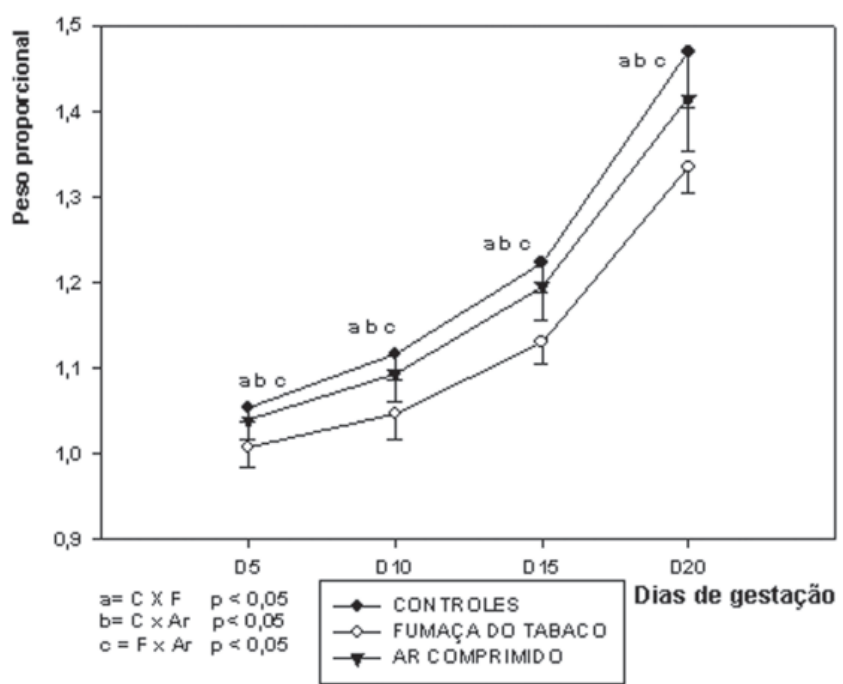

Figura 2 - Valores médios e desvios-padrão de peso proporcional ao peso da cópula durante a gestação, de ratas expostas à fumaça do tabaco $(F)$ e ao ar comprimido ( $\mathrm{Ar}$ ) comparadas aos controles (C) (D5 - quinto dia; D10 - décimo dia; D15 - décimo-quinto dia; D20 - vigésimo dia). 
O grupo F consumiu significantemente menos alimento por dia em média que o grupo ar comprimido $(p<0,05)$ e o grupo controle $(p<0,001)$. O grupo ar comprimido, por sua vez, também apresentou consumo alimentar médio menor que o grupo controle $(\mathrm{p}<0,05)$ [Grupo $\mathrm{F}=18,9( \pm 1,2)$; Grupo $\mathrm{Ar}=21,7$ $( \pm 1,6)$; Grupo $\mathrm{C}=24,2( \pm 1,7)]$ (Figura 3).

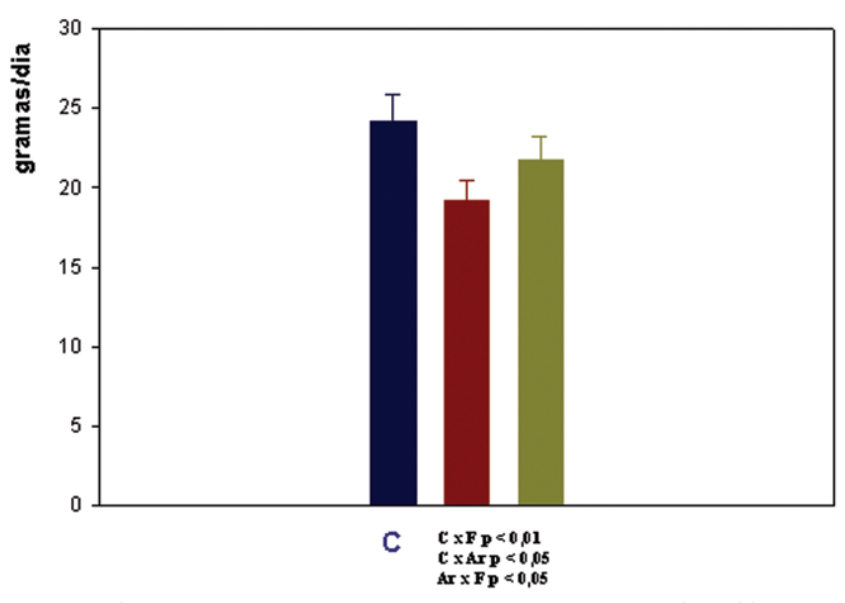

Figura 3 - Consumo alimentar médio em gramas por dia, medido do $1^{\circ}$ ao $21^{\circ}$ dias de gestação, de ratas expostas à fumaça do tabaco $(\mathrm{F})$ e ao ar comprimido (Ar), comparadas a controles (C).

A Tabela 1 mostra a média e desvio padrão do peso e do comprimento dos filhotes das ratas estudadas. Nota-se que os filhotes de ratas expostas ao tabaco tiveram menor peso e comprimento ao nascimento em relação aos grupos ar comprimido e controle, e que esses dois últimos grupos não diferiram entre si. Os valores médios para os pesos dos filhotes por grupo foram: peso: Grupo $\mathrm{F}=5,5 \mathrm{~g}( \pm 0,3)$ vs Grupo Ar=5,9 g $( \pm 0,5)$ vs Grupo $\mathrm{C}=5,9 \mathrm{~g}( \pm 0,4)(\mathrm{p}<0,01)$; quanto ao comprimento: Grupo $F=6,8 \mathrm{~cm}( \pm 0,2)$ vs Grupo $\operatorname{Ar}=6,9 \mathrm{~cm}( \pm 0,2) \times$ Grupo $\mathrm{C}=6,9 \mathrm{~cm}( \pm 0,1)(\mathrm{p}<0,05)$.

Tabela 1 - Média e desvio padrão do peso $(\mathrm{g})$ e do comprimento $(\mathrm{cm})$ dos filhotes das ratas ao nascer, nos 3 grupos experimentais.

\begin{tabular}{lccc}
\hline $\begin{array}{l}\text { Grupos } \\
\text { experimentais }\end{array}$ & $\mathbf{n}$ & $\begin{array}{c}\text { Peso }(\mathbf{g}) \\
\overline{\mathbf{x}} \pm \mathbf{D P}\end{array}$ & $\begin{array}{c}\text { Comprimento }(\mathbf{c m}) \\
\overline{\mathbf{x}} \pm \mathbf{D P}\end{array}$ \\
\hline Fumaça do tabaco & 15 & $5,5 \pm 0,3^{*}$ & $6,8 \pm 0,2^{*}$ \\
Ar comprimido & 18 & $5,9 \pm 0,5$ & $6,9 \pm 0,1$ \\
Controles & 18 & $5,9 \pm 0,4$ & $6,9 \pm 0,1$ \\
${ }^{*} \mathrm{p}<0,05$. & & &
\end{tabular}

\section{Discussão}

Este estudo mostra que o grupo de ratas expostas ao tabaco, quando comparado ao grupo de animais controle, obteve menor ganho ponderal desde o início da gestação. O seguimento do peso materno, iniciado a partir da constatação da prenhez, mostrou que o prejuízo no ganho ponderal ocorre precocemente no grupo de ratas expostas à fumaça lateral de cigarros. O mesmo efeito foi observado por meio da análise do peso proporcional ao peso da cópula, que desconta o efeito do peso de entrada do animal no estudo.

A exposição ao ar comprimido, isoladamente, também foi capaz de prejudicar o ganho absoluto de peso das ratas gestantes quando comparadas aos controles no final da gestação. Além disso, o peso proporcional em relação ao peso na cópula foi menor em todos os períodos da gestação, o que sugere que a manipulação dos animais, por si só, foi capaz de prejudicar o ganho ponderal das ratas. Descontado o efeito da manipulação, a comparação do grupo fumante com o grupo ar comprimido mostrou que o cigarro, isoladamente, também prejudicou o ganho ponderal materno em todo o período do estudo. Esta diferença foi mais nítida quando se descontou o peso de entrada de cada animal no experimento (peso proporcional), confirmando que as diferenças encontradas entre os animais fumantes e controles não tinham ocorrido apenas devido à manipulação, e sim à exposição à fumaça do cigarro.

É conhecido que a exposição experimental à fumaça do tabaco reduz o ganho ponderal de ratas na gravide $z^{31,32}$. Experimentos que procuraram analisar o efeito da composição da fumaça do tabaco mostraram que a exposição à fumaça de outra origem vegetal teve efeito menor sobre o ganho ponderal de ratas grávidas que a fumaça do tabaco, mesmo quando se adicionou nicotina ${ }^{33}$. Também a exposição ao monóxido de carbono puro levou a redução menos notável do ganho ponderal que a exposição à fumaça completa do tabaco com concentrações comparáveis deste gás ${ }^{31,32}$.

Em estudo realizado em ratas, empregandose injeções com doses variando de 0,5 a $5,0 \mathrm{mg} /$ $\mathrm{kg}$ de nicotina, duas vezes ao dia, observou-se que quanto maior a dosagem empregada, menor o incremento de consumo alimentar observado na gestação e menor o incremento de peso em relação a animais controle que receberam injeção de solução salina ${ }^{34}$. Os animais que foram apenas manipulados (receberam injeções de solução salina) apresentaram também redução de consumo alimentar em relação aos controles, mas em menor proporção que as ratas que receberam injeções de nicotina.

Mecanismo responsável pelo menor ganho ponderal das ratas gestantes expostas à fumaça lateral de cigarros seria o efeito anorético do tabaco. Estudo utilizando o mesmo sistema de expo- 
sição à fumaça do tabaco, observou redução do consumo alimentar, do ganho ponderal e do crescimento de ratos jovens ${ }^{29}$. Outro estudo experimental conduzido com o objetivo de verificar o efeito do fumo e da nicotina sobre o consumo alimentar mostrou redução significativa do consumo alimentar, com conseqüente diminuição do ganho de peso nos animais ${ }^{35}$.

No presente estudo, o consumo alimentar do grupo exposto ao tabaco foi reduzido durante todo o período estudado, comparado aos controles. A manipulação dos animais isoladamente foi capaz de prejudicar o consumo alimentar e conseqüentemente o ganho ponderal das ratas na gestação. Assim, parte dos prejuizos observados no ganho de peso das ratas pode ser atribuída à manipulação dos animais (exposição ao ar comprimido), porém o efeito deletério no ganho de peso materno foi agravado pela exposição ao tabaco e, possivelmente, pela diminuição da ingestão alimentar das ratas que, por sua vez, poderia ser responsabilizada pelas alterações metabólicas características da exposição ao tabaco (causada por inúmeras substâncias presentes no cigarro), que atuaram no decorrer da gestação.

No grupo de ratas expostas ao fluxo de ar comprimido, a manipulação foi capaz de comprometer o ganho ponderal absoluto e relativo das ratas gestantes, devido à redução significativa do consumo alimentar, sem comprometer o crescimento intrauterino dos filhotes.

A comparação dos resultados do grupo ar comprimido do presente estudo, com outros relatos de modelos de estresse é dificultada pela heterogeneidade das técnicas empregadas. Modelos de estresse aplicados a ratas gestantes têm mostrado comprometimento do peso dos filhotes $^{36,37}$, com variação no comprometimento do peso materno ${ }^{36-38}$.

A restrição alimentar em ratas gestantes não foi capaz de prejudicar o peso dos filhotes tanto quanto a exposição tabágica ${ }^{32}$. Assim, ratas que consumiram quantidades semelhantes de dieta às consumidas pelas ratas fumantes apresentaram redução de peso nos seus filhotes em relação a animais controles, porém o efeito da exposição ao cigarro foi mais acentuado que o da redução proposital da dieta isoladamente. Quando se comparou o efeito da exposição ao cigarro e da exposição ao monóxido de carbono, mantendo fixa a ingestão energética, observou-se que, apesar de as mães ganharem peso na gravidez de formas semelhantes, os filhotes do grupo exposto ao cigarro apresentavam menor peso ao nascimento. Este achado sugere que a transferência de nutrientes das mães para os fetos via placenta tenha sido negativamente afetada pelo tabagismo, possivelmente devido a alterações morfológicas e funcionais da placenta.

O mecanismo fisiopatológico da redução de peso de filhotes de ratas submetidas à exposição à fumaça lateral de cigarros durante a gestação é provavelmente o mesmo aventado para humanos, ou seja, hipóxia fetal originada por duas vias independentes, porém, aditivas: o efeito agudo da liberação de catecolaminas induzido pela nicotina que resulta em hipóxia fetal episódica e ocorre devido a vasoconstricção materna e perfusão uterina reduzida, e o aumento prolongado dos niveis de carboxiemoglobina fetal, resultando em hipoxemia fetal persistente ${ }^{17,18,39}$. Além disso, modificações morfológicas maternas e placentárias poderiam resultar em efeitos hormonais e tróficos sobre o feto ${ }^{40}$.

No presente estudo, a comparação de peso dos filhotes realizada ao nascimento mostrou que o peso foi menor no grupo de filhotes de ratas expostas à fumaça de cigarros, em relação aos demais grupos. Além disso, o prejuízo de crescimento intra-uterino observado nos filhotes das ratas expostas ao tabaco foi independente da manipulação, visto que os animais apenas manipulados e os do grupo controle não apresentaram diferença no peso e estatura ao nascer entre si. Estes achados de redução de peso e comprimento ao nascimento de filhotes de ratas expostas à fumaça lateral de cigarros estão de acordo com o observado em outros estudos experimentais ${ }^{29,34}$.

Os resultados do presente estudo permitem concluir que o ganho de peso e o consumo alimentar de ratas gestantes foram afetados pela exposição ao tabaco, e que parte deste efeito é devido ao estresse da manipulação, uma vez que as ratas manipuladas e submetidas apenas ao fluxo de ar comprimido também apresentaram redução de peso em relação aos animais controle. No entanto, apenas os filhotes das ratas expostas ao tabaco apresentaram redução de peso e do comprimento analisados ao nascimento.

\section{Referências}

1. Lieberman E, Gremy I, Lang JM, Cohen AP. Low birthweight at term and the timing of fetal exposure to maternal smoking. Am J Public Health. 1994;84(7):1127-31.

2. Cnattingius S, Haglund B. Decreasing smoking prevalence during pregnancy in Sweden: the effect on small-for-gestational-age births. Am J Public Health. 1997;87(3):410-3. 
3. Dejmek J, Solansky I, Podrazilova K, Sram RJ. The exposure of nonsmoking and smoking mothers to environmental tobacco smoke during different gestational phases and fetal growth. Environ Health Perspect. 2002;110(6):601-6.

4. Magee BD, Hattis D, Kivel NM. Role of smoking in low birth weight. J Reprod Med. 2004;49(1):23-7.

5. Chiolero A, Bovet P, Paccaud F. Association between maternal smoking and low birth weight in Switzerland: the EDEN study. Swiss Med Wkly. 2005;135(35-36):525-30.

6. Schechter MD, Cook PG. Nicotine-induced weight loss in rats without an effect on appetite. Eur J Pharmacol. 1976;38(1):63-9.

7. Falkeborn Y, Larsson C, Nordberg A. Chronic nicotine exposure in rat: a behavioural and biochemical study of tolerance. Drug Alcohol Depend. 1981;8(1):51-60.

8. Perkins KA. Weight gain following smoking cessation. J Consult Clin Psychol. 1993;61(5):76877 .

9. Hofstetter A, Schutz Y, Jequier E, Wahren J. Increased 24-hour energy expenditure in cigarette smokers. N Engl J Med. 1986;314(2):79-82.

10. Perkins KA. Effects of tobacco smoking on caloric intake. Br J Addict. 1992;87(2):193-205.

11.Lemos-Santos MGF, Gonçalves-Silva RMV, Botelho C. Tabagismo, composição corporal, distribuição da adiposidade e ingestão alimentar em fumantes, não fumantes e ex-fumantes. Folha Méd. 2000; 119(3):23-31.

12. Hall SM, Ginsberg D, Jones RT. Smoking cessation and weight gain. J Consult Clin Psychol. 1986;54(3):342-6.

13. Perkins KA, Sexton JE, DiMarco A, Fonte C. Acute effects of tobacco smoking on hunger and eating in male and female smokers. Appetite. 1994; 22(2):14958.

14.Zaren B, Lindmark G, Gebre-Medhin M. Maternal smoking and body composition of the newborn. Acta Paediatr. 1996;85(2):213-9.

15. Horta BL, Kramer MS, Platt RW. Maternal smoking and the risk of early weaning: a meta-analysis. Am J Public Health. 2001;91(2):304-7.

16. Horta BL, Victora CG, Menezes AM, Halpern R, Barros FC. Low birthweight, preterm births and intrauterine growth retardation in relation to maternal smoking. Paediatr Perinatal Epidemiol. 1997;11(2):140-51.

17. Lymperopoulou A, Hainaut F, Crimal PH, Durand $\mathrm{JL}$, Locatelli C, Maison C. Tabac et grossesse: recherche d'une corrélation cotininémie et Doppler. J Gynecol Obstet Biol Reprod (Paris). 1996;25(8):8247.
18. Pinto GR, Botelho C. Influência do tabagismo no sistema vascular materno-fetal: estudo com dopplervelocimetria. Rev Bras Ginecol Obstet. 2000;22(10):641-6.

19. Weiss ST, Tager IB, Schenker M, Speizer FE. The health effects of involuntary smoking. Am Rev Respir Dis. 1983;128(5):933-42.

20. Scherer G, Conze C, von Meyerinck L, Sorsa M, Adlkofer F. Importance of exposure to gaseous and particulate phase components of tobacco smoke in active and passive smokers. Int Arch Occup Environ Health. 1990;62(6):459-66.

21. Law RL, Hackshaw AK. Environmental tobacco smoke. Br Med Bull. 1996;52(1): 22-34.

22. Sopori ML, Kozak W. Immunomodulatory effects of cigarette smoke. J Neuroimmunol. 1998;83(1-2):14856

23. Centro Latinoamericano de Perinatología y Desarrollo Humano (CLAP). Tabaquismo y embarazo: hay que ayudar a parar. Salud Perinat. 1987;2(7):65-77.

24. Chen LH, Petitti DB. Case-control study of passive smoking and the risk of small-for-gestational-age at term. Am J Epidemiol. 1995;142(2):158-65.

25. Witschi H, Joad JP, Pinkerton KE. The toxicology of environmental tobacco smoke. Annu Rev Pharmacol Toxicol. 1997;37:29-52.

26. Windham GC, Eaton A, Hopkins B. Evidence for an association between environmental tobacco smoke exposure and birthweight: a meta-analysis and new data. Paediatr Perinat Epidemiol. 1999;13(1):35-57.

27. Nafstad P, Fugelseth D, Qvigstad E, Zahlsen K, Magnus P, Lindemann R. Nicotine concentration in the hair of nonsmoking mothers and size of offspring. Am J Public Health. 1998;88(1):120-4.

28. Mello PRB. Efeito do tabagismo sobre a liberação de prolactina, produção láctea e componentes imunes do leite de ratas [tese]. São Paulo: Universidade de São Paulo; 2004.

29. Gonçalves-Silva RMV, Lemos-Santos MG, Botelho C. Influência do tabagismo no ganho ponderal, crescimento corporal, consumo alimentar e hídrico de ratos. J Pneumol. 1997;23(3):124-30.

30. Mello PRB, Okay TS, Dores EFGC, Botelho C. Avaliação de um sistema de exposição tabágica passiva em modelo experimental utilizando cotinina e carboxiemoglobina como marcadores de exposição. Pulmão RJ. 2005;14(3):228-36.

31. Tachi N, Aoyama M. Effect of cigarette smoke and carbon monoxide inhalation by gravid rats on the conceptus weight. Bull Environ Contam Toxicol. 1983;31(1):85-92.

32. Tachi N, Aoyama M. Effect of restricted food supply to pregnant rats inhaling carbon monoxide on fetal 
weight, compared with cigarette smoke exposure. Bull Environ Contam Toxicol. 1986;37(6):877-82

33. Younoszai MK, Peloso J, Haworth JC. Fetal growth retardation in rats exposed to cigarette smoke during pregnancy. Am J Obstet Gynecol. 1969;104(8):1207-13.

34. Becker RF, Little CR, King JE. Experimental studies on nicotine absorption in rats during pregnancy. 3. Effect of subcutaneous injection of small chronic doses upon mother, fetus, and neonate. Am J Obstet Gynecol. 1968;100(7):957-68.

35. Grunberg NE. Nicotine, cigarette smoking, and body weight. Br J Addict. 1985;80(4):369-77.

36. Stilmann Salgado A, Maris Martinez S, Cristina Tarres M. Body weight of litters of rats stressed during pregnancy. Medicina (B Aires). 1977;37(1):3842 .
37. Cabrera RJ, Rodríguez-Echandía EL, Jatuff AS, Foscolo M. Effects of prenatal exposure to a mild chronic variable stress on body weight, preweaning mortality and rat behavior. Braz J Med Biol Res. 1999;32(10):1229-37.

38. Billinson MR. Prematurity and low birth weight litters: a mechanism elicited by thermal stress. Am J Obstet Gynecol. 1970;108(6):970-4.

39. Viggiano MG, Caixeta AM, Barbacena ML. Fumo e gravidez: repercussões sobre concepto e placenta. J Bras Ginecol. 1990;100(7):147-52.

40. Czekaj P, Palasz A, Lebda-Wyborny T, NowaczykDura G, Karczewska W, Florek E, et al. Morphological changes in lungs, placenta, liver and kidneys of pregnant rats exposed to cigarette smoke. Int Arch Occup Environ Health. 2002;75 Suppl:S27-35. 